maintenance of cardio-renal function in liver failure and may indicate that albumin plays a crucial role in moderating inflammation.

Competing interests None declared.

\section{OC-069 ROTATIONAL THROMBOELASTOMETRY IN CIRRHOSIS: HYPERCOAGULABLE AND HYPERFIBRINOLYTIC}

doi:10.1136/gutjnl-2012-302514a.69

${ }^{1} \mathrm{~V}$ Jairath, ${ }^{2} \mathrm{D}$ Hall, ${ }^{3} \mathrm{P}$ Harrison, ${ }^{4} \mathrm{~S}$ Stanworth, ${ }^{2} \mathrm{~T}$ Walsh, ${ }^{5} \mathrm{P}$ Hayes, ${ }^{1} \mathrm{~J}$ Collier, ${ }^{4} \mathrm{M}$ Murphy, ${ }^{6}$ E Barnes. ${ }^{1}$ John Radcliffe Hospital, Oxford, UK; ${ }^{2}$ Critical Care, Edinburgh Royal Infirmary, Edinburgh, UK; ${ }^{3}$ Oxford Haemophilia and Thrombosis Centre, UK; ${ }^{4} \mathrm{NHS}$ Blood and Transplant, Oxford, UK; ${ }^{5}$ Department of Hepatology, Edinburgh Royal Infirmary, Edinburgh, UK; ${ }^{6}$ Department of Hepatology, John Radcliffe Hospital, Oxford, UK

Introduction Cirrhotics have complex acquired derangements of haemostasis. Routine coagulation tests suggest a hypocoagulable profile, resulting in frequent administration of blood components for prophylaxis and treatment of bleeding. Rotational thromboelastography $\left(\right.$ ROTEM $\left.^{\circledR}\right)$, unlike standard coagulation tests, provides a real-time measurement of clot formation, strength and stability in whole blood and may more accurately reflect in vivo coagulation. We aimed to (1) identify the key derangements in the haemostatic pathways in patients with cirrhosis; (2) determine the prevalence of overt hyperfibrinolysis and whether this could be improved with anti-fibrinolytics.

Methods We used ROTEM ${ }^{\circledR}$ to investigate: (1) Clotting time (CT) and maximum clot firmness (MCF) in stable, non-bleeding cirrhotics compared to healthy volunteers; (2) The presence of overt hyperfibrinolysis and whether this could be reversed by spiking blood samples ex vivo with the antifibrinolytic aprotinin (APTEM test). Overt hyperfibrinolysis was defined by a maximum lysis (ML) of $>15 \%$ and by comparing the clot lysis index at 60 min between EXTEM and APTEM parameters.

Results 106 adult cirrhotics and 28 healthy volunteers were enrolled after informed consent. Median EXTEM CT was shorter in cirrhotics than controls (51s vs $58 \mathrm{~s}, \mathrm{p}<0.01)$ and the clotting time shortened as Child-Pugh score increased in severity (52s Child A, 49s, Child B, 47s Child $\mathrm{C}$ ). In cirrhotics there was strong correlation between EXTEM MCF with both platelet count $(\mathrm{r}=0.801, \mathrm{p}<0.0001)$ and fibrinogen levels $(r=0.653, p<0.0001)$, as well as fibrinogen and FIBTEM MCF $(\mathrm{r}=0.641, \mathrm{p}<0.0001) .25 \%(26 / 106)$ of cirrhotics had evidence of overt hyperfibrinolysis. After spiking samples from cirrhotics with aprotinin, hyperfibrinolysis was completely reversed $(\mathrm{ML}<15 \%)$ in $50 \%$ $(13 / 26)$ cases and partially reversed in 50\% (13/26) cases. There was a significant reduction in the median ML between EXTEM and APTEM clot profiles (13 vs 11, $\mathrm{p}<0.001)$. D-dimer levels increased with increasing disease severity (Child A-894, Child B-1835, Child C-5281). Conclusion Cirrhotics have a hypercoagulable clotting time, despite prolonged PT, APTT and thrombocytopenia supporting the concept of re-balanced haemostasis. Use of ROTEM ${ }^{\circledR}$ may avoid unnecessary and potentially harmful transfusion of pro-coagulant blood components in cirrhotics. The high prevalence of overt hyperfibrinolysis in cirrhosis requires further elucidation and clinical studies to investigate the potential role of anti-fibrinolytics in the prophylaxis of variceal bleeding.

Competing interests None declared.

\section{BAPEN symposium: "original communications" OC-070 SYSTEMATIC REVIEW ON THE PREVALENCE OF MALNUTRITION IN GENERAL PRACTICE}

doi:10.1136/gutjn-2012-302514a.70

${ }^{1} \mathrm{P}$ McGurk, ${ }^{*} \mathrm{~A}$ Cawood, ${ }^{2,3} \mathrm{R}$ Stratton, ${ }^{3} \mathrm{M}$ Elia. ${ }^{1}$ Nutrition and Dietetics, University Hospital Southampton NHS Trust, Southampton, UK; ${ }^{2}$ Medical Affairs, Nutricia,
Trowbridge, UK; ${ }^{3}$ Institute of Human Nutrition, University of Southampton, Southampton, UK

Introduction Information on the prevalence of malnutrition in general practice is very limited. This review was undertaken to establish the overall prevalence of malnutrition among adult patients registered with General Practitioners (GP), and also in specific patient groups.

Methods A literature search was undertaken using standard systematic review methodology on 22 August 2011 using Pub Med (1948-2011), Embase (1980-2011) Cochrane Collaboration (2011) and also by cross referencing. Studies were included if they involved adults $\geq 18$ years, registered at a GP in any country provided they reported the prevalence of malnutrition. They were excluded if the populations were not linked to GP or involved pregnant or lactating women.

Results Of 54 studies initially identified, only seven met the inclusion criteria (five from the UK). Five set out to establish the prevalence of malnutrition (group 1) but another two reported prevalence (group 2). In group 1 studies the prevalence of malnutrition ranged widely, $0 \%-12 \%$, with up to $38 \%$ being at risk of malnutrition. They involved different methodologies (consecutive patients, random sampling, subjects from larger studies, GP databases) and heterogeneous populations, varying in age ( $\geq 18$ years $(\mathrm{n}=2$ studies), $\geq 65$ years $(\mathrm{n}=2)$, and $>90$ years $(\mathrm{n}=1))$ and diagnosis/clinical status (Diabetes $(n=1)$, chronic diseases including cancer and CVD $(n=1)$, no "acute illness" ( $n=1)$, "apparent wellness" $(n=1)$ and a mixture of health and various diseases $(n=1))$. They identified malnutrition using Mini Nutritional Assessment $(n=2)$, Body Mass Index (BMI) with varying cut-offs $\left(\leq 18.5 \mathrm{~kg} / \mathrm{m}^{2}, \mathrm{n}=1\right.$; $\left.<20 \mathrm{~kg} / \mathrm{m}^{2}, \mathrm{n}=1\right)$, and unintentional weight loss $(\mathrm{n}=1)$. Among group 2 studies, one reported a prevalence of only $0.4 \%$ in people aged $\geq 65$ years, according to entries about malnutrition in the GP notes extracted from a large randomly selected population ( $n=75$ 176) from the General Practice Research Database. ${ }^{1}$ The other group 2 study reported a prevalence of $0 \%$ among a randomly selected group of subjects $\geq 75$ years using mid-arm circumference, mid-upper arm muscle circumference and triceps skinfold thickness that were less than the 5th centile of reference data of a very different population group.

Conclusion This systematic review confirms that there is a lack of data on the prevalence of malnutrition in general practice, with relevant studies using different identification methods and populations, making it difficult to establish a reliable overall prevalence.

Competing interests None declared.

\section{REFERENCE}

1. Margolis DJ, Knauss J, Bilker W, et al. Medical conditions as risk factors for pressure ulcers in an outpatient setting. Age Ageing 2003;32:259-64.

\section{OC-071 WITHDRAWN}

